\title{
Transición del concepto de industrias creativas y su papel en el entorno económico
}

Transition of the concept of creative industries and its role in the economic environment

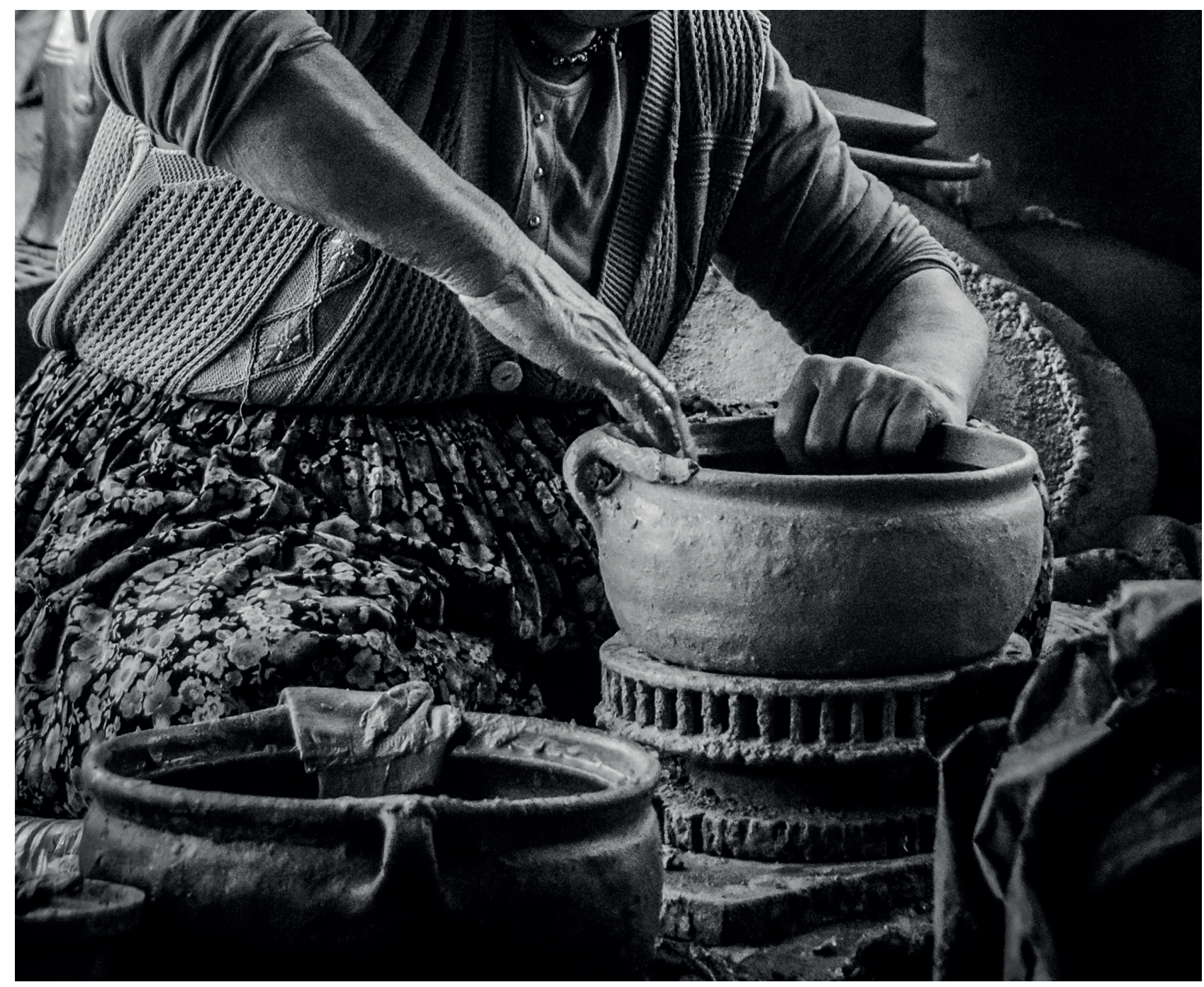




\title{
Transición del concepto de industrias creativas y su papel en el entorno económico' \\ Transition of the concept of creative industries and its role in the economic environment
}

\section{David Juliao-Esparragoza², Mariangela Lopez-Lambraño³, Alberto Muñoz-Santiago ${ }^{4}$}

Artículo recibido en junio 25 de 2020; artículo aceptado en septiembre 11 de 2020

\begin{abstract}
Este artículo puede compartirse bajo la Licencia Creative Commons Atribución-NoComercial-Compartirlgual 4.0 Internacional y se referencia usando el siguiente formato: Juliao-Esparragoza, D., Lopez-Lambraño, M. y Muñoz-Santiago, A. (2021). Transición del concepto de industrias creativas y su papel en el entorno económico. I+D Revista de Investigaciones, 16(1), 225-237. http://dx.doi.org/10.33304/ revinv.v16n1-2021018.
\end{abstract}

\begin{abstract}
Resumen
Este artículo de reflexión hace un recorrido por los conceptos de industrias culturales y creativas. Muestra la transición de los términos desde su visión cultural y artística hasta el entendimiento del papel de dichas industrias en el ecosistema económico. Su propósito estriba en el entendimiento de estas industrias como eje de desarrollo a partir de una visión política clara, con direccionamientos estratégicos identificados. Se realiza un análisis bibliométrico básico que permite identificar la importancia que tienen las industrias creativas en la actualidad, y también se hace una aproximación de su impacto a través de indicadores económicos mundiales y una lectura de la eficiencia de estas en la región. Se identifican los retos asociados a la industria y se proponen alternativas de abordaje en el contexto colombiano.
\end{abstract}

Palabras clave: Creatividad, economía naranja, innovación, desarrollo económico, reflexión.

\begin{abstract}
This reflection paper makes a journey through the concepts of cultural and creative industries. It shows the transition of these terms from their cultural-artistic vision to the understanding of the role of industries in the economic ecosystem. Its purpose is the understanding of this industrial sector as the axis of development of a clear political view with strategic identified directions. A bibliometric analysis was done to identify the importance of creative industries at present, and an approximation of its impact is made through global economic indicators and the reading of the efficiency of the industries in the region. The challenges of the industry are identified and alternative approaches are proposed in the Colombian context.
\end{abstract}

Keywords: Creativity, orange economy, innovation, economic development, reflection.

\footnotetext{
${ }^{1}$ Artículo de reflexión, de diseño cualitativo, perteneciente al área de ciencias sociales, subárea de administración y negocios, desarrollado en el grupo de investigación Innovar, Universidad del Norte (Barranquilla, Colombia). Dirección: km 5 vía Puerto Colombia. PBX: +57 (5) 3509509 . Fecha de inicio: septiembre 2019. Fecha de terminación: agosto 2020.

2 Economista, Universidad del Atlántico. Magíster en Administración de Empresas, Universidad del Norte. Docente-investigador del grupo GIM. Universidad del Norte (Barranquilla, Colombia). Dirección: km 5 vía Puerto Colombia, PBX: (+57) (5) 3509509. ORCID ID: https://orcid.org/00000002-4142-3873. Correo electrónico institucional: djuliae@uninorte.edu.co.

${ }^{3}$ Ingeniero civil, Universidad del Norte. Magíster en Administración de Empresas, Universidad del Norte. Docente-investigador del grupo Innovar. Universidad del Norte (Barranquilla, Colombia). Dirección: km 5 vía Puerto Colombia, PBX: (+57) (5) 3509509. ORCID ID: https://orcid.org/00000001-7802-5935. Correo electrónico institucional: mlambrano@uninorte.edu.co.

${ }^{4}$ Administrador de Empresas, Universidad del Norte. Magíster en Economía Empresarial, Incae. Docente-investigador del grupo Innovar. Universidad del Norte (Barranquilla, Colombia). Dirección: km 5 vía Puerto Colombia, PBX: (+57) (5) 3509509. ORCID ID: https://orcid.org/0000-0001-9821-0940. Correo electrónico institucional: amunoz@uninorte.edu.co.
} 
David Juliao-Esparragoza, Mariangela Lopez-Lambraño, Alberto Muñoz-Santiago Transición del concepto de industrias creativas y su papel en el entorno económico

\section{Introducción}

La globalización a la que están expuestos los países hoy en día los obliga a que busquen ventajas competitivas que les permitan ser sostenibles a lo largo del tiempo, de forma que puedan avanzar en la ruta de estrategias que ofrezcan productos y servicios con un claro y diferenciado valor agregado a sus consumidores más allá de su valor económico.

Existen tendencias muy fuertes que proponen la búsqueda de alternativas diferentes. Es por eso por lo que algunos países, especialmente en América Latina, están apostando por los conceptos de economías creativas y culturales para potencializar su desarrollo económico.

América Latina es una región rica en componentes culturales, con matices étnicos muy propios y arraigados que permiten una diversidad cultural muy amplia. Por tal motivo no le resultará difícil incursionar en las nuevas propuestas de economía creativa y cultural, haciendo de ellas su principal ventaja competitiva como región. Para lograr lo anterior es importante destinar grandes esfuerzos y recursos dirigidos a fomentar e incentivar espacios creativos en las ciudades y zonas rurales que ayuden a potencializar las economías creativas (AcostaMedina et al., 2019).

Las ciudades que han focalizado parte de sus estrategias políticas y económicas en temas como la creatividad son capaces de ofrecer soluciones a la gran cantidad de problemas que las aquejan, lo que da origen a procesos de transformación urbana con un sello cultural importante. Este tipo de ciudades se vuelven atractivas para personas emprendedoras, inversionistas, turistas, para nuevas empresas, entre otras, capaces de valorar y apreciar el potencial que estas ofrecen (Boix y Lazzeretti, 2011; Pose García, 2015).

Para los países de América Latina, las economías creativas representan una oportunidad única de potencialización de sus recursos a partir de la cultura, creatividad e innovación. Para esto se necesitan grandes esfuerzos e iniciativas en la formalización empresarial de los proyectos que le apuestan a este tipo de iniciativas. (Bustamante, 2009). Si bien algunos autores han enfocado esfuerzos en el entendimiento del fenómeno de industrias creativas (Podestá y Hernández, 2012), este ejercicio, a juicio de los autores del presente trabajo, representa un acercamiento más a los tránsitos académicos del concepto en relación con su uso y transformaciones, con lo que aportan elementos para fomentar el debate. Se entiende este ejercicio como un esfuerzo de caracterización y reflexión sobre industrias creativas, especialmente en entornos latinoamericanos y específicamente en Colombia, donde los esfuerzos por dinamizar la industria han sido tímidos (González Vélez, 2013).
Para lograr esto se parte de una revisión general sobre cómo el concepto ha evolucionado a nivel teórico y luego se pone atención a reflexiones generales de indicadores relacionados con su dinámica, retos y oportunidades.

\section{Metodología}

\section{Tipo de estudio}

El tipo de estudio presentado es exploratorio, su alcance es de tipo reflexivo y su intención es exponer a partir de una caracterización de la evolución del concepto industrias creativas un marco para su análisis en los contextos latinoamericanos. El ejercicio de evolución del concepto se culmina con un ejercicio básico a nivel bibliométrico, con análisis de coocurrencias y reflexiones derivadas para consolidar la economía creativa como un elemento de desarrollo en Colombia.

\section{Procedimiento}

El ejercicio de aproximación bibliométrica se adelantó con el soporte del software de versión libre Vos Viewer. Se tomó con fuente secundaria el Core Collection de Web of Science, utilizando la ventana de tiempo de 10 años. El indicador clave de búsqueda fue "Creative Industries". Se registraron los datos en términos de la frecuencia en el tiempo, los tipos de contribución, los países de origen y los autores más citados. Por último, se corrió en el software un análisis de coocurrencia de palabras que permitió identificar núcleos de discusión donde orbitan o convergen los trabajos académicos sobre el tema de búsqueda.

\section{Resultados}

\section{Definición de industrias culturales e industrias creativas}

Los términos "industrias culturales" e "industrias creativas" son una categoría reciente en la discusión académica (Cunningham, 2002). Son diversas las fuentes citadas en la literatura que remiten a los conceptos seminales. Uno de estos viene de los esfuerzos del Reino Unido mediante la iniciativa liderada por el Primer Ministro Tony Blair en 1997 cuando creó el Grupo de trabajo de industrias creativas del Reino Unido (The U.K. Creative Industries Task Force). Este grupo hizo posible un mapeo claro de las industrias creativas en esta parte de Europa, poniendo énfasis en los núcleos de la actividad, sus características descriptivas y su impacto en la economía (Department for Digital, Culture, Media and Sport, 1998).

Otros autores identifican que en la descripción del término "industria cultural" se deben identificar no solo los mercados y productos básicos que de ella se derivan, sino entender el término en la complejidad de relaciones que implica, intentando descubrir los factores que influyen en sus limitaciones y factores de 
expansión (Hesmondhalgh, 2008). También es entendido como una extensión de mercados sociales (Potts et al., 2008). Así, la transformación del término "industria de la cultura", que era vista principalmente desde el marginalismo de transacciones de orden decorativo que atendían mercados basados en el hedonismo, comenzó a visualizarse dentro de la formulación de políticas como potencial recurso económico (O'Connor, 2010).

Son múltiples las referencias que destacan la importancia de la cultura en el contexto de la gestión de negocios (Tian et al., 2018), especialmente como un factor crítico en el desarrollo internacional (Verspagen, 2006), con claras influencias en las dinámicas nacionales de innovación (Shane, 1993).
Una vez que el concepto de industria cultural fue resignificado desde su aparición inicial con los trabajos de Max Horkheimer y Adorno en la década de los cuarenta, tomó fuerza su visión como sector productivo, y se crearon las condiciones para que en la década de los noventa se llegara a un tránsito del concepto de "industria cultural" al concepto de "industrias creativas", este último con un anclaje claro en la explotación de la propiedad intelectual. Es un término más amplio que incluye no solo las artes, sino también un conjunto diversificado de bienes tangibles e intangibles con contenido creativo y alto valor para el mercado (Szpilbarg y Saferstein, 2014).

Por otra parte, son muchas las acepciones que desde organismos multilaterales se le han dado a la "economía creativa". Algunos de estos se resumen en la Tabla 1.

Tabla 1

Definiciones relacionadas con "economía creativa - industrias culturales y creativas"

\begin{tabular}{|c|c|}
\hline Organización & Definición \\
\hline $\begin{array}{l}\text { Organización de las Naciones Unidas } \\
\text { para la Educación, la Ciencia y la Cultura } \\
\text { (UNESCO) (2010) }\end{array}$ & $\begin{array}{l}\text { Las industrias culturales y creativas son una fusión entre bienes tangibles o intangibles de } \\
\text { concepción creativa que gozan de la protección de derechos legales (protección de derechos de } \\
\text { autor); incluyen, entre otras, la publicidad y arquitectura. }\end{array}$ \\
\hline $\begin{array}{l}\text { Conferencia de las Naciones Unidas sobre } \\
\text { Comercio y Desarrollo (UNCTAC) (2010) }\end{array}$ & $\begin{array}{l}\text { Las industrias creativas orbitan en la concepción de economías creativas. Se entienden como } \\
\text { procesos en cuya dinámica se encuentran como componentes la creatividad y el capital } \\
\text { intelectual. Son clasificadas de diferentes formas incluyendo su utilidad como patrimonio, medios } \\
\text { artísticos y creaciones funcionales. }\end{array}$ \\
\hline $\begin{array}{l}\text { Organización Mundial de la Propiedad } \\
\text { Intelectual (OMPI) (2005) }\end{array}$ & $\begin{array}{l}\text { Las industrias resguardadas o cubiertas por derechos de autor son aquellas que se relacionan de } \\
\text { forma directa o indirecta con capitales intelectuales protegidos en toda la cadena. }\end{array}$ \\
\hline $\begin{array}{l}\text { Department for Digital, Culture, Media } \\
\text { and Sport (2001) }\end{array}$ & $\begin{array}{l}\text { Las industrias creativas tienen como epicentro las competencias individuales que promueven la } \\
\text { propiedad intelectual. Tienen influencia directa en la dinámica económica a través de empleo y } \\
\text { riqueza. }\end{array}$ \\
\hline $\begin{array}{l}\text { Comisión Económica para América Latina } \\
\text { y el Caribe (NU. CEPAL, 2014) }\end{array}$ & $\begin{array}{l}\text { Teniendo en cuenta la definición dada por la Comisión Económica para América Latina y el Caribe } \\
\text { (CEPAL), las industrias creativas de contenidos se definen como las actividades relacionadas con } \\
\text { actividades editoriales, cine, televisión, radio, discográfica, contenidos para celulares, producción } \\
\text { audiovisual, independiente; contenidos para páginas web, videojuegos, y contenidos relacionados } \\
\text { con la convergencia digital (cross media). }\end{array}$ \\
\hline $\begin{array}{l}\text { Banco Interamericano de Desarrollo } \\
\text { (Benavente y Grazzi, 2017) }\end{array}$ & $\begin{array}{l}\text { Se entiende las industrias culturales como aquellas que ofrecen productos y servicios de } \\
\text { orientación cultural reflejados en las políticas de cada región. }\end{array}$ \\
\hline
\end{tabular}

Fuente: Autores.

Las acepciones del término tienen como eje común la labor de explotación del capital intelectual y artístico. Se promueven en las definiciones los acercamientos a derechos de autor como parte complementaria e importante y la explotación con fines comerciales de la creación de productos (tangibles o intangibles). Se acercan algunas definiciones a tipologías específicas donde el deporte, los servicios creativos y la convergencia digital son piedras angulares en la clasificación.

\section{Actividades económicas asociadas a las industrias creativas}

La economía creativa plantea una visión diferente a la economía tradicional; la economía creativa es más flexible y destaca una relación especial en toda la cadena de suministros. Según Howkins (2002), el contenido de la economía creativa va desde las artes hasta campos como la ciencia y la tecnología, e incluye las actividades económicas presentadas en la Tabla 2.

Tabla 2

Actividades económicas

\begin{tabular}{ccc}
\hline $\begin{array}{c}\text { Arte, por ejemplo: la } \\
\text { pintura }\end{array}$ & Artesanía & Diseño \\
Moda & Cine & Música \\
$\begin{array}{c}\text { Artes escénicas: } \\
\text { teatro, ópera, danza } \\
\text { y ballet }\end{array}$ & $\begin{array}{c}\text { Edición y } \\
\text { publicaciones de } \\
\text { libros y revistas } \\
\begin{array}{c}\text { Programas de } \\
\text { cómputo }\end{array}\end{array}$ & $\begin{array}{c}\text { Juguetes y juegos, } \\
\text { excluyendo los } \\
\text { videojuegos. } \\
\text { Videojuegarollo }\end{array}$ \\
\hline Arquitectura & Televisión y radio. \\
\hline
\end{tabular}

Fuente: Howkins (2002). 
David Juliao-Esparragoza, Mariangela Lopez-Lambraño, Alberto Muñoz-Santiago Transición del concepto de industrias creativas y su papel en el entorno económico

Se puede evidenciar, a partir de las diferentes aproximaciones teóricas, que el concepto de economía creativa ha mutado desde visiones individuales a conceptos más abstractos que incluyen los efectos de esta industria en la sociedad; así mismo, la dinámica académica basada en un análisis bibliométrico básico del concepto permite ver su importancia como objeto de estudio.

\section{Análisis bibliométrico básico del concepto de industrias creativas}

\section{Hallazgos por área de conocimiento}

Teniendo en cuenta los 1041 registros obtenidos en Web of Science con el criterio de búsqueda "Creative
Industries", se observa que el término tiene asiento principalmente en administración, geografía, estudios culturales, economía y estudios ambientales, donde cada una de estas áreas mantiene registros superiores a 120 publicaciones. La distribución de registros se muestra a continuación: administración, 213 registros (20\%); geografía, 180 (17\%); estudios culturales, 171 (16\%); economía, 145 (13\%), y estudios ambientales, 125 (12\%).

Se observa que la disciplina que mayor dinamismo ha mostrado en términos de las publicaciones anuales del concepto de industrias creativas es administración, seguida por geografía, estudios culturales y economía. Las primeras áreas que comprenden registros sobre industrias creativas en el año 2001 fueron administración y economía. En el 2003 se unió geografía y solo hasta 2008, estudios culturales (ver Figura 1).

\section{Número de artículos sobre industrias creativas registrados por área de conocimiento (2001-2020)}

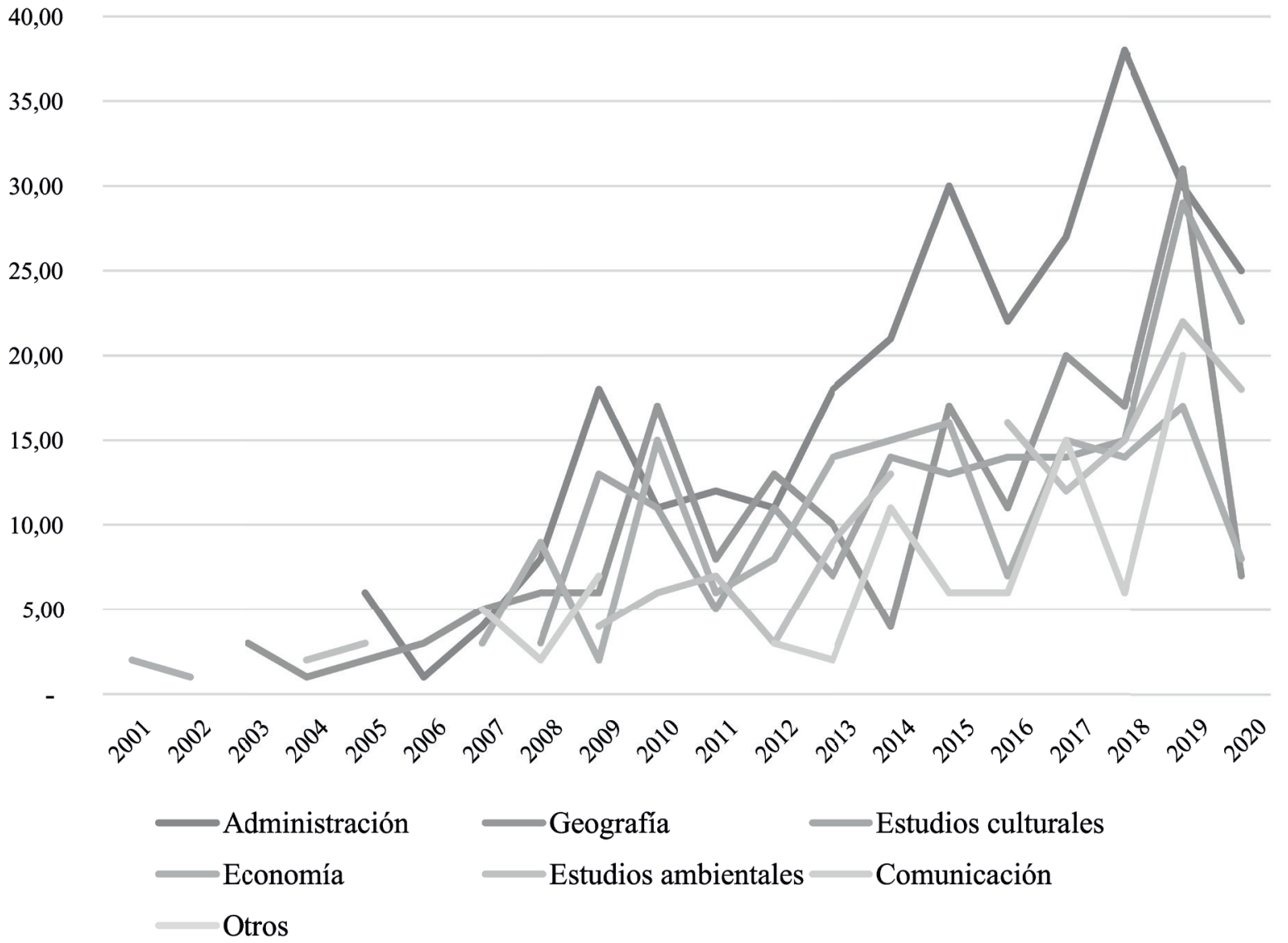

Figura 1. Número de artículos sobre industrias creativas registrados por área de conocimiento (2001-2020). Fuente: Autores, a partir de Web of Science. 
David Juliao-Esparragoza, Mariangela Lopez-Lambraño, Alberto Muñoz-Santiago

Transición del concepto de industrias creativas y su papel en el entorno económico

Crecimiento de referentes académicos sobre industrias creativas en la ventana de tiempo

El primer registro obtenido de la base de datos Web of Science en la ventana de tiempo 2001-2020 empieza en el 2001. Al séptimo año el número de artículos publicados se triplicó. Hasta la primera década se publicaron 190 artículos. Se evidencia un punto de inflexión en el año 2017, donde se llega a una cifra superior a los 100 registros por año, cifra que se mantiene en promedio el siguiente año y alcanza un pico importante en 2019, al registrar 149 publicaciones, lo que evidencia un dinamismo o interés de publicación en el tema (ver Figura 2).

\section{Publicaciones sobre industrias creativas (2001-2020)}

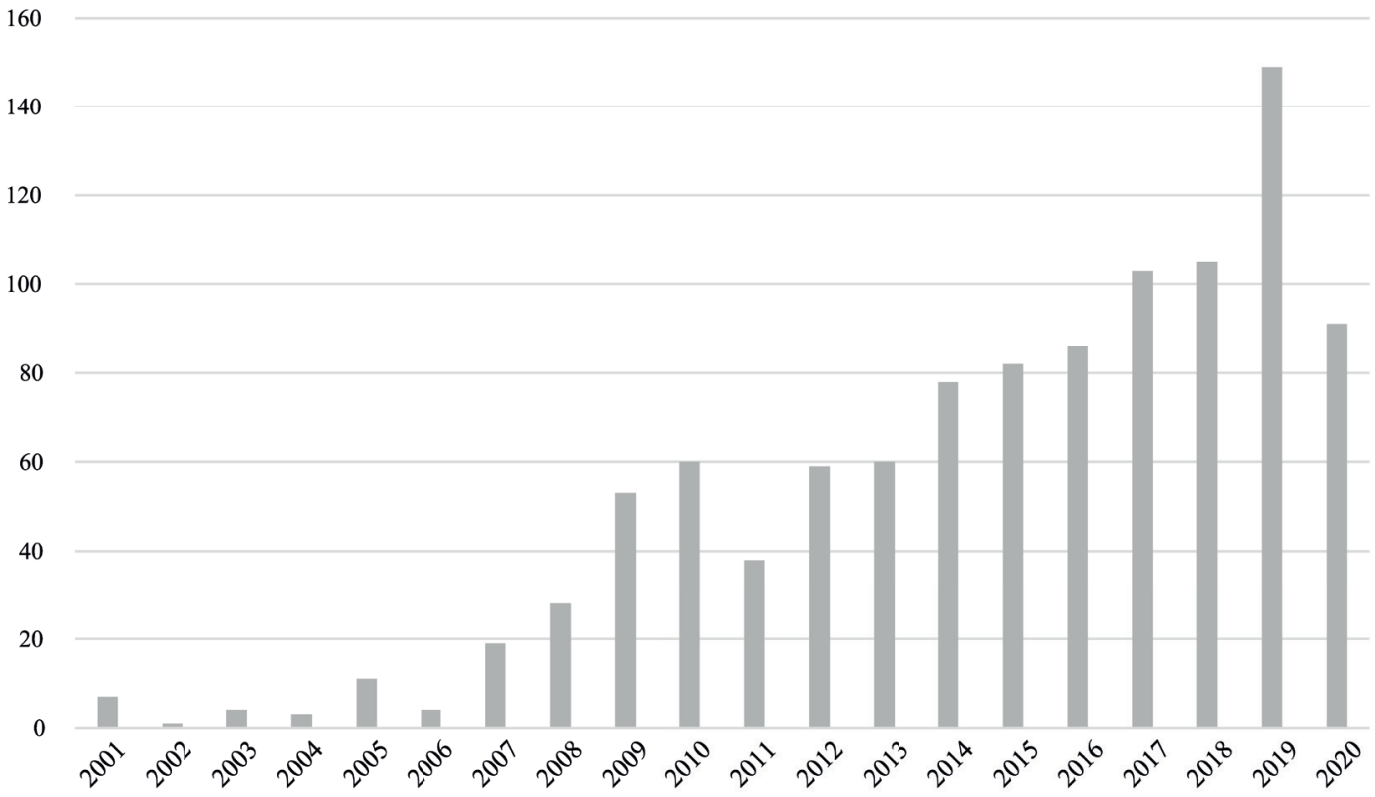

Figura 2. Publicaciones sobre industrias creativas (2001-2019). Fuente: Autores, con base en Web of Science.

\section{Registros por tipo de documento}

Como se observa en la Tabla 3, se destaca claramente que el tipo de documento escogido para publicar sobre industrias creativas es el artículo científico, seguido por reseña de libros: 901 y 94, respectivamente.

Tabla 3

Registros de contribución por tipo de documento

\begin{tabular}{cc}
\hline Tipo de documento & Cantidad \\
\hline Article & 901 \\
Book review & 94 \\
Early access & 30 \\
Editorial material & 24 \\
Review & 21 \\
Book chapter & 3 \\
Proceedings paper & 12 \\
Meeting abstract & 1 \\
\hline
\end{tabular}

Fuente: Autores, a partir de datos de Web of Science.

\section{Registros por país de procedencia}

Se observa en el Figura 3 que los países de procedencia de la mayor parte de las contribuciones intelectuales son Inglaterra y Australia. En general estos países mantienen una dinámica de publicaciones cuya proporción es 2:1, con respecto a países como Estados Unidos y Alemania. Es importante señalar que el único país de América del Sur es Brasil.

\section{Autores más citados}

os 954 registros obtenidos han sido citados 6226 veces, con un promedio de cita por artículo de 6,53. Las publicaciones más citadas se muestran en la Tabla 4. 
David Juliao-Esparragoza, Mariangela Lopez-Lambraño, Alberto Muñoz-Santiago

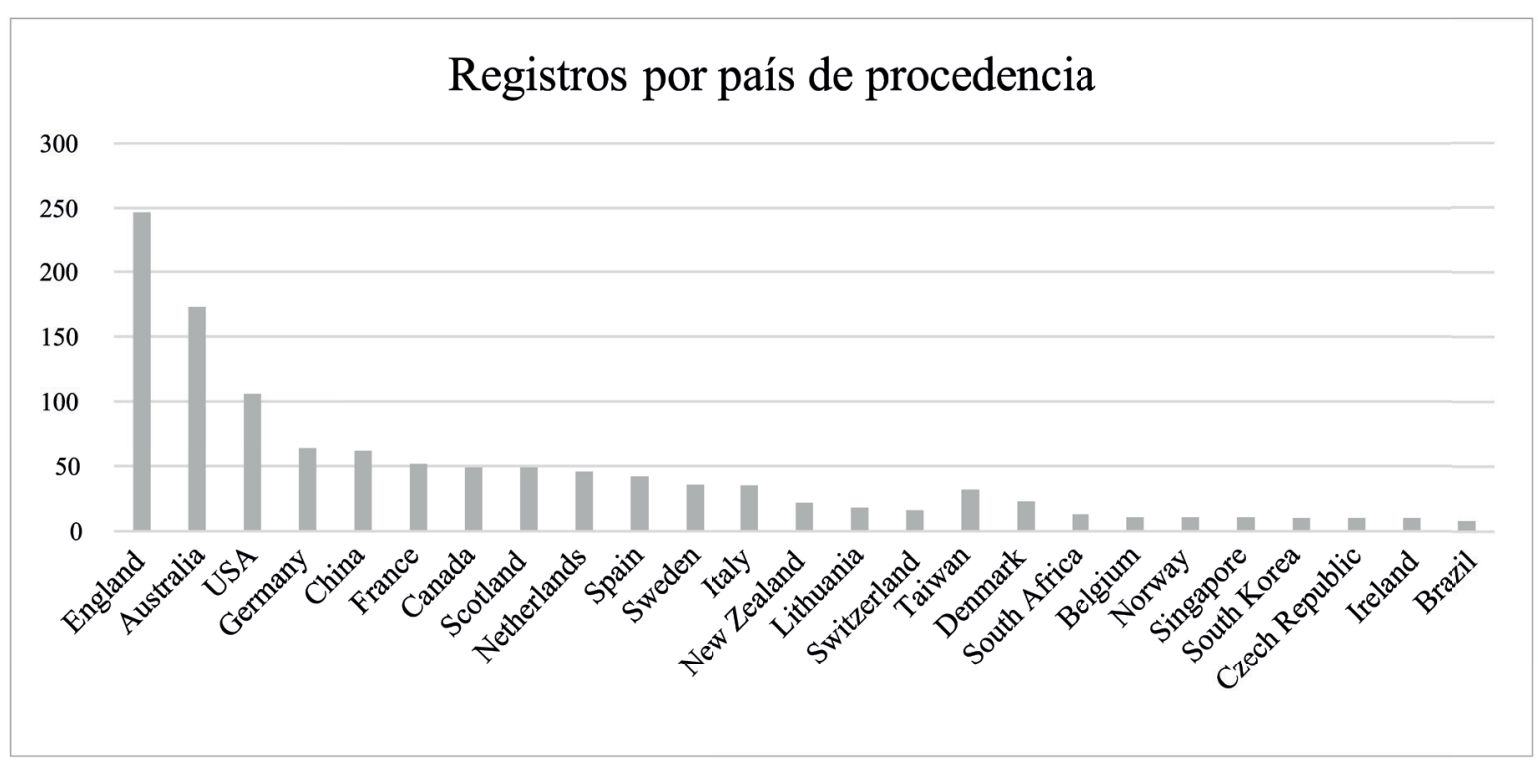

Figura 3. Registros por país de procedencia. Fuente: Autores, a partir de Web of Science.

Tabla 4

Citas recibidas por autor

\begin{tabular}{|c|c|c|c|}
\hline Trabajo & Autores & $\begin{array}{c}\text { Total de citas } \\
2001-2019\end{array}$ & $\begin{array}{c}\text { Promedio por } \\
\text { año }\end{array}$ \\
\hline Creative cities: The cultural industries and the creative class & Pratt, Andy C. & 263 & 21,92 \\
\hline $\begin{array}{l}\text { A core/periphery perspective on individual creative performance: } \\
\text { Social networks and cinematic achievements in the Hollywood } \\
\text { film industry }\end{array}$ & Cattani, Gino; Ferriani, Simone. & 212 & 17,67 \\
\hline $\begin{array}{l}\text { 'This place gives me space': place and creativity in the creative } \\
\text { industries }\end{array}$ & Drake, G. & 181 & 10,65 \\
\hline $\begin{array}{l}\text { Social network markets: a new definition of the creative indus- } \\
\text { tries }\end{array}$ & $\begin{array}{l}\text { Potts, Jason; Cunningham, Stuart; } \\
\text { Hartley, John; Ormerod, Paul }\end{array}$ & 176 & 14,67 \\
\hline Looking for work in creative industries policy & Banks, Mark; Hesmondhalgh, David & 142 & 12,91 \\
\hline $\begin{array}{l}\text { Small businesses in the new creative industries: innovation as a } \\
\text { people management challenge }\end{array}$ & Hotho, Sabine; Champion, Katherine & 137 & 15,22 \\
\hline $\begin{array}{l}\text { Defining the creative economy: Industry and occupational } \\
\text { approaches }\end{array}$ & $\begin{array}{l}\text { Markusen, Ann; Wassail, Gregory H.; } \\
\text { DeNatale, Douglas; Cohen, Randy }\end{array}$ & 129 & 10,75 \\
\hline $\begin{array}{l}\text { Creative imitation: exploring the case of cross-industry innova- } \\
\text { tion }\end{array}$ & Enkel, Ellen; Gassmann, Oliver & 123 & 12,3 \\
\hline $\begin{array}{l}\text { Do creative industries cluster? Mapping creative local production } \\
\text { systems in Italy and Spain }\end{array}$ & $\begin{array}{l}\text { Lazzeretti, Luciana; Boix, Rafael; Capo- } \\
\text { ne, Francesco }\end{array}$ & 119 & 9,92 \\
\hline $\begin{array}{l}\text { Polic transfer as policy assemblage: making policy for the creative } \\
\text { industries in New Zealand }\end{array}$ & Prince, Russell & 107 & 10,7 \\
\hline
\end{tabular}

Fuente: Autores, a partir de Web of Science.

\section{Análisis de coocurrencias "por palabra (co-word)"}

El análisis de coocurrencia de palabras es muy útil para identificar los temas analizados en los artículos, ya que se supone que las palabras con mayor mención en un documento aportan una descripción del contenido, así como vínculos entre temas y otros artículos.

Mediante el software VOS Viewer, que analizó la información ingresada, se construyó el mapa de análisis de relación de palabras (ver Figura 4). Se identificaron 5 núcleos asociados a palabras (innovación, creatividad, ciudades, economía, conocimiento). Cada esfera representa una palabra, y su tamaño representa la ocurrencia de esta dentro del conjunto de artículos; las líneas varían en grosor dependiendo de la relación entre palabras. El proceso de filtro de la información para la creación del mapa basado en datos de texto consistió en configurar al software VOS Viewer para que extrajera de la base de datos los títulos y los resúmenes de los 954 artículos más relevantes; se seleccionó un umbral de mínimo 4 ocurrencias por palabra. Del total de palabras, 100 cumplían la condición establecida. 


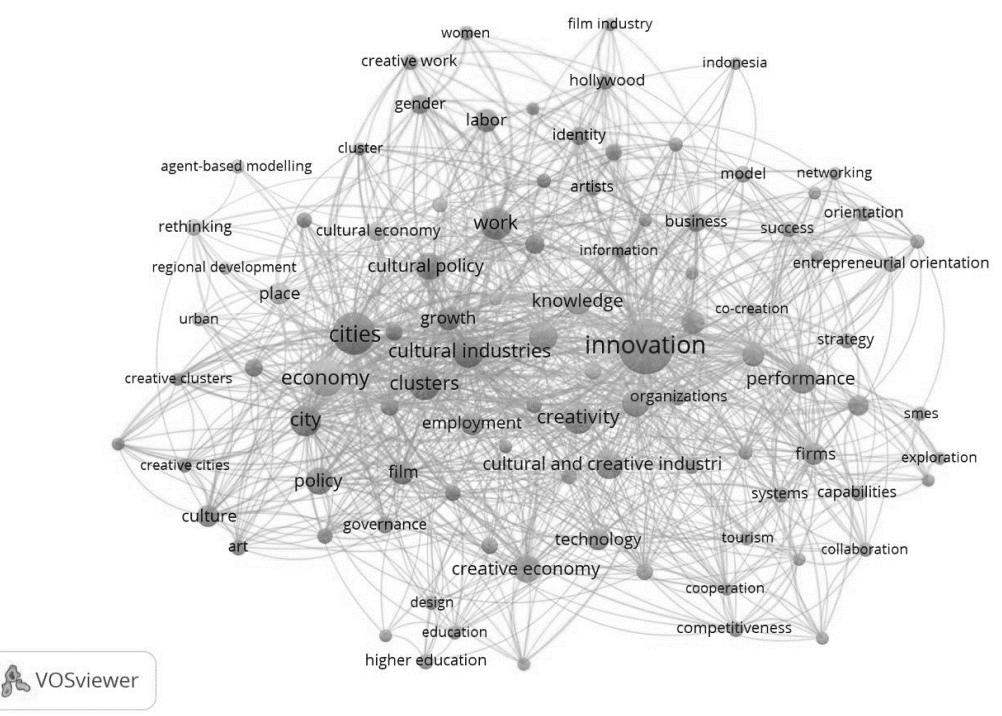

Figura 4. Mapa de palabras: Coocurrencia. Fuente: Autores utilizando la herramienta Vos Viewer.

El término con mayor ocurrencia es "innovación"; el segundo término con mayor repetición en los artículos es "ciudades", y lo siguen "economía" y "desempeño".

La contribución académica tiene foco en la forma en que las industrias creativas generan valor a la sociedad. Los términos performace (desempeño) y ciudades se conectan toda vez que los artículos dejan ver esta relación. De hecho, para Pratt (2008) la contribución de mayor importancia de la industria es el papel de la producción cultural en el crecimiento económico de las ciudades. Así mismo los lugares o los atributos de las localidades pueden incentivar procesos creativos en una relación de "clusters creativos". Las conexiones evidenciadas en el Figura 5 lo confirman; lo anterior es conforme a lo expresado por Drake (2003).
Sin embargo, si bien las localidades pueden favorecer la creatividad como un mecanismo que promueve mejores absorciones, existen evidencias de autores que expresan su disconformidad con el tratamiento normativo de estos espacios. Se evidencia que algunos países han mostrado renuencia a reconocer problemas de explotación, inseguridad y desigualdad, que se evidencian en estas industrias y que podrían generar trabas en la dinámica de su crecimiento (Banks y Hesmondhalgh, 2009).

Otra visualización importante en el mapa de coocurrencias es cómo el concepto ha venido variando en el tiempo y hacia dónde están migrando las palabras claves conforme a los años de aparición.

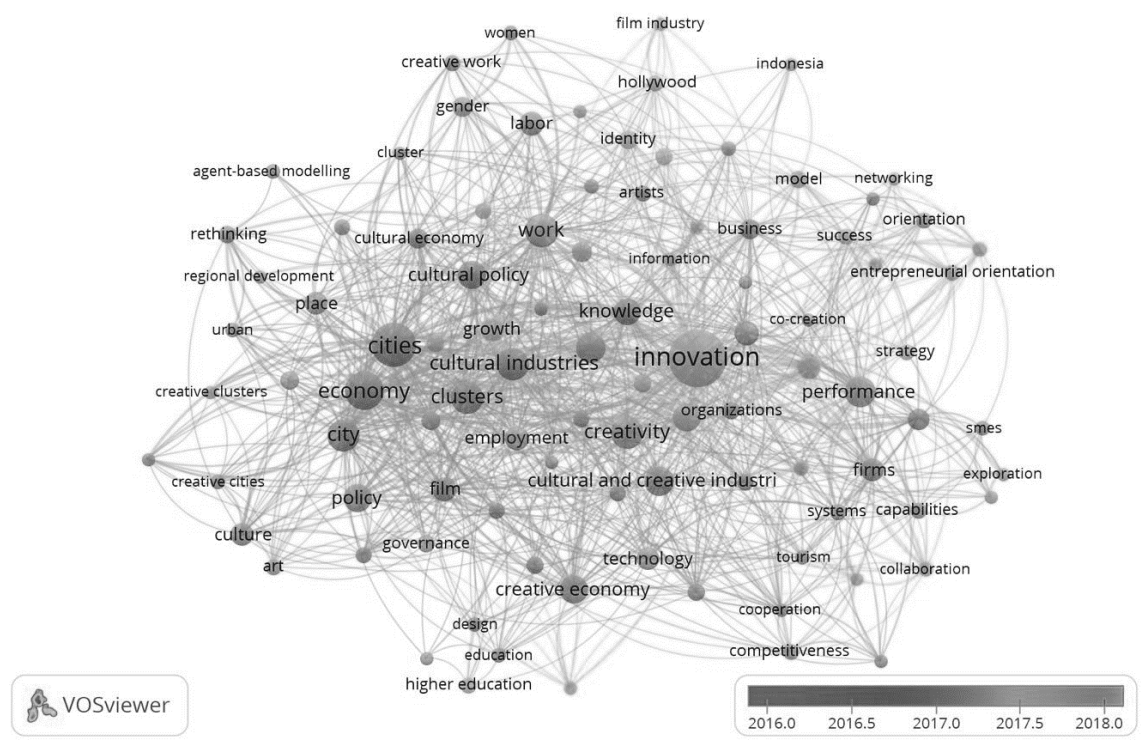

Figura 5. Mapa de palabras: Coocurrencia (en el tiempo). Fuente: Autores utilizando la herramienta Vos Viewer. 
David Juliao-Esparragoza, Mariangela Lopez-Lambraño, Alberto Muñoz-Santiago Transición del concepto de industrias creativas y su papel en el entorno económico

Se observa en el Gráfico 5 que si bien el término tenía como anclaje primario los temas de trabajo, empleo y economía (2016), el tránsito hacia ciudades, cluster y desempeño fueron mostrándose como nuevos nodos (2017). Para 2018 aparecen como nuevas asociaciones la colaboración y la orientación hacia el emprendimiento. En este contexto puede evidenciarse cómo ha sido la evolución y el impacto de las industrias creativas en el escenario mundial.

\section{Ingresos generados por las ICC (industrias creativas) en todo el mundo}

En el ámbito mundial la industria creativa generó 2,25 billones de dólares en ingresos durante el 2013. Esta suma supera en un $43 \%$ los ingresos del sector de las telecomunicaciones y en un $18 \%$ el PIB de la India. Los sectores más representativos en relación con los ingresos de la ICC son televisión, artes visuales y periódicos y revistas, con una participación del $21 \%$, $17 \%$ y $16 \%$, respectivamente. Analizando la participación en la generación de empleo por parte de la industria, se estima que en 2013 existía 29,5 millones realizando actividades relacionadas con la creatividad y la cultura. Esta cifra es un $18 \%$ más de los empleos generados por las telecomunicaciones, y un $11 \%$ más que el empleo en la India. Los sectores que más generaron empleo en la industria creativa fueron artes visuales, libros y música, con el $29 \%, 12 \%$ y $13,5 \%$ de los empleos de la economía naranja para el 2013.

La Tabla 5 resume el desarrollo de las regiones en términos de generación de ingresos y empleo (Organización de las Naciones Unidas para la Educación, UNESCO, y Confederación Internacional de Sociedades de Autores y Compositores, CISAC, 2015).

Tabla 5

Ingresos de la industria creativa a nivel mundial

\begin{tabular}{|c|c|c|c|}
\hline & Ingresos & Empleo & Ingresos/Empleo \\
\hline Total mundial & $\begin{array}{l}2,25 \\
\text { billones de } \\
\text { dólares }\end{array}$ & $29,5 \mathrm{MM}$ & $\begin{array}{l}\text { US\$ } 76.271 \text { por perso- } \\
\text { na empleada }\end{array}$ \\
\hline Asia Pacífico & $\begin{array}{l}73.000 \mathrm{MM} \\
\text { de dólares }\end{array}$ & $12,7 \mathrm{MM}$ & $\begin{array}{c}\text { US\$ } 58.503 \text { por perso- } \\
\text { na empleada }\end{array}$ \\
\hline Europa & $\begin{array}{l}709.000 \\
\text { MM de } \\
\text { dólares }\end{array}$ & 7,7 MM & $\begin{array}{l}\text { US\$ } 92.078 \text { por perso- } \\
\text { na empleada }\end{array}$ \\
\hline Norteamérica & $\begin{array}{l}620.000 \\
\text { MM de } \\
\text { dólares }\end{array}$ & 4,7 MM & $\begin{array}{c}\text { US\$ } 131.915 \text { por perso- } \\
\text { na empleada }\end{array}$ \\
\hline Latinoamérica & $\begin{array}{l}124.000 \\
\text { MM de } \\
\text { dólares }\end{array}$ & 1,9 MM & $\begin{array}{l}\text { US\$ } 65.263 \text { por perso- } \\
\text { na empleada }\end{array}$ \\
\hline $\begin{array}{l}\text { África Medio y } \\
\text { Oriente }\end{array}$ & $\begin{array}{l}58.000 \mathrm{MM} \\
\text { de dólares }\end{array}$ & 2,4 MM & $\begin{array}{c}\text { US\$ } 24.166 \text { por perso- } \\
\text { na empleada }\end{array}$ \\
\hline
\end{tabular}

Fuente: Autores con datos del Informe Tiempo de Culturas (2015).
En la Tabla 5 se evidencian las diferencias significativas en productividad del trabajo entre las diferentes regiones. Es la norteamericana la que encabeza este ranking, duplicando la productividad de Latinoamérica y multiplicando seis veces la de África, sin duda debido al avance en cine, televisión y a los desarrollos de cultura digital, mientras que los africanos fundamentan su desarrollo en la ICC en una industria cinematográfica mal estructurada.

\section{Estrategias para consolidar a la economía creativa como elemento dedesarrollo en Colombia}

El término 'creativo' transmite un mensaje de tratarse de una actividad que no está sometida a ningún tipo de regla o condicionamiento, lo que en el fondo lleva a pensar que es informal, $y$, por lo tanto, carente de sistemas de medición serios y con pocas posibilidades de estructurarse como un área estratégica para el gobierno de cualquier país. Bajo este supuesto, la mayor parte de los países han menospreciado su importancia como generador de desarrollo.

A pesar de que durante años se ha hablado del tema, existen muy pocos casos donde se observe una estructura clara y formal, como para cualquier otro sector de la economía, y por consiguiente pocas mediciones de su importancia y mucho menos un plan estructurado para su proyección. Esto hace que el análisis para evaluar su evolución se convierta en una actividad parecida a armar un rompecabezas donde muchas piezas faltan.

Analizando esta situación, es necesario que en nuestro país, sobre todo, se implemente una política que permita cuantificar el impacto de la ICC en la economía, sobre todo en ingresos y empleos de forma sostenida y amplia, ya que si bien existen algunos esfuerzos (Cámara de Comercio de Barranquilla, 2016; Ministerio de Cultura de Colombia-Convenio Andrés Bello, 2003), estos son en la mayoría aislados y no permiten análisis longitudinales. Los autores sugieren de forma respetuosa algunos lineamientos de apoyo a estas industrias:

\section{Fomentar el cooperativismo como estrategia de desarrollo de la industria creativa: innovar en los} canales de comercialización. El Gobierno debe crear mecanismos que fomenten la asociación en esta industria con miras a obtener desarrollos más significativos en términos de volumen y calidad. Un mecanismo sería la posibilidad de desarrollar canales digitales de promoción y comercialización, tales como redes, cluster, cooperativas, asociaciones, etc., tomando en cuenta que la digitalización permite llegar a un mercado más grande en tiempo real. En la industria del software y del juego, sin duda una asociación estratégica genera ventajas competitivas para los participantes y actúa como un mecanismo de apoyo en los procesos de medición de resultados en el sector. 
David Juliao-Esparragoza, Mariangela Lopez-Lambraño, Alberto Muñoz-Santiago

Transición del concepto de industrias creativas y su papel en el entorno económico

2. Fomentar el espíritu emprendedor y de formalización de los artistas: los actores principales. Solo en la medida en que el mercado general observe que la ICC se formaliza en el país, empezará a darle importancia. Aunque en el país existen subsectores con aparente grado de formalización, aun la gran masa se mueve en la informalidad. Diseñar un plan de capacitación y estímulo para que el artista se convierta en emprendedor es el primer paso para la formalización general del sector. Dicho estímulo debe incluir algunas exenciones de tipo tributario, créditos blandos, ayudas a la exportación, etc. Tales medidas rendirían frutos palpables a favor de la formalización del sector. Pero es un proceso donde el artista debe ser líder, y solo lo será si encuentra beneficios tales como la visibilidad de su producción y, por supuesto, mejoras en su calidad de vida.

3. Fomentar la participación activa de la mujer en el proceso de digitalización de la industria creativa. Estudios de todo tipo en el ámbito muestran que de manera gradual la mujer está obteniendo mayor participación como ofertante y demandante en el mercado laboral, o sea, tiene mayor participación en la economía, no solo en niveles operativos, sino también en niveles directivos en las organizaciones. La ICC no puede ser una excepción; se deben llevar a cabo esfuerzos para que la mujer participe en el proceso de modernización del sector, y así pueda asumir el liderazgo que le ha sido negado o impedido en épocas anteriores y genere o impulse su ingreso en el mundo de las TIC, ya sea para el desarrollo de productos o simplemente para su promoción y venta.

4. Incentivar la cultura y tradición como eje central de la estrategia creativa. Una de las características en otras regiones del mundo (la norteamericana y la europea) es sustentar el desarrollo de la ICC en sus culturas y tradiciones. Si analizamos el cine, Norteamérica en sus inicios se basó en aspectos relacionados con su historia desde el momento de la colonización hasta las guerras mundiales; sus contenidos en general tratan de dirigir la atención del consumidor hacia resaltar sus tradiciones, mientras en Europa sucede igual. Lo anterior sin duda sustenta la necesidad de sentar las bases del sector en aspectos culturales colombianos: carnavales de tradición, Festival Vallenato, mitos y leyenda, etc., no solo en aspectos de su desarrollo vernáculo, sino como contenido de todas las áreas que componen la ICC. Toda la tecnología moderna en el país debería dirigirse a mantener las tradiciones y mostrarlas, lo cual permitirá en la era digital que las personas de otras partes del mundo las vivencien sin necesidad de viajar.

5. Crear mercados regionales para las industrias creativas. La generación de marcas país o marcas región inicia el proceso de posicionamiento de los productos creativos. Es necesario para esto que, en términos generales, se creen estructuras físicas y virtuales para dar visibilidad y comercializar los productos, generar identidad de estos y de alguna forma crear presión de grupo ante los posibles compradores, creando oportunidades que permitan cerrar brechas existentes, que son evidentes sobre todo en entornos emergentes (Rotondo y Legonía Córdova, 2018). Es necesario crear mercados regionales que permitan a las empresas de la ICC tener mayor conectividad con el mercado potencial a través de ferias, muestras o simplemente dando visibilidad virtual a los productos. Sin duda, exponer la región de manera digital tendrá como consecuencia de mediano plazo el incremento del turismo hacia esta.

6. Establecer líneas de fomento financiero para la creación de empresas ICC. El Gobierno, mediante la creación de esquemas de financiamiento amigables, canales de inversión y redes de protección social, incentivará la formalización en la ICC. Los ingresos por ventas en esta industria son variables e inciertos, por lo que un esquema proteccionista al inicio permitirá disminuir el riesgo asociado a su operación. Sin duda, debe buscarse la creación de un banco de fomento a favor de esta industria o permitir que los actuales puedan de manera viable financiar el crecimiento de sus empresas. Adicionalmente, deben diseñarse programas de capacitación a los creativos en aspectos relacionados con la estrategia, la operación y el manejo financiero de sus negocios.

7. Utilizar la preservación de la naturaleza como insumo inspirador para la industria creativa. Desde la antigüedad el hombre ha encontrado en la naturaleza (adicionalmente a la cultura) una fuente de inspiración para sus creaciones y una fuente de insumos para el diseño de sus productos. Solo una política seria en relación con el manejo medioambiental permitirá un crecimiento sostenido de las ICC. El uso dañino del recurso por parte de esta y otras industrias, además de ir contra la misma existencia humana, va en contra de su propia estabilidad operativa. Hoy tanto científicos como biólogos y ambientalistas cumplen un papel fundamental con vistas al futuro de toda la humanidad y el mantenimiento de sus esquemas de vida.

8. Generar una reglamentación sólida y democrática sobre la protección intelectual en la ICC. El Gobierno debe buscar los mecanismos para hacer públicas las bases de datos de las creaciones registradas en el país. Ello implica necesariamente facilitar los procesos de registros y protección de las creaciones por medio de procesos ágiles y transparentes que conviertan la defensa de la autoría en algo sumamente sencillo, pero que a la vez proteja a quien registra una invención o creación artística.

9. Dirigir los rendimientos económicos del sector
al mismo sector. Un mecanismo para proteger la 
David Juliao-Esparragoza, Mariangela Lopez-Lambraño, Alberto Muñoz-Santiago Transición del concepto de industrias creativas y su papel en el entorno económico

industria e incentivar la formalización consiste en que el emprendedor de la ICC observe que, a través de su desarrollo, se incrementa la calidad de vida de todos los participantes en este sector de la economía.

10. La cuantificación del impacto creativo. Los gobiernos deben estructurar esquemas novedosos que permitan medir el impacto de desarrollos simples, tales como una nueva danza, una canción, un libro o cualquier otra creación de la ICC en una región o país. Se trata de un proceso complejo, por lo cual los sistemas de medición deben diseñarse de manera consensuada con los creativos, para no sobreestimar o subvalorar cualquier creación. Es un hecho real el enorme grado de informalidad que muestra el mercado laboral de la ICC, por lo que en la región es más complejo aun diseñar un modelo de medición. Se requiere, por lo tanto, implementar inicialmente un proceso de formalización del trabajo creativo, ya que con esta sería más fácil establecer indicadores, como número de empleos en la industria creativa, indicadores de productividad y rentabilidad $y$, sobre todo, darle el estatus de importancia que requiere la actividad de artista profesional (Finlev et al., 2017).

\section{Conclusiones}

La economía creativa tiene un vínculo muy estrecho con el sector público y con el sector informal, lo cual le da una connotación especial. Por tal motivo, su gestión requiere un tratamiento especial que sea transversal a diversos factores políticos, culturales y comerciales, entre otros (Quartesan et al., 2007).

Es necesario plantear enfoques diferentes a los tradicionales, teniendo en cuenta a los productores, a los consumidores y a las redes que impulsan la innovación. Lo anterior es muy diferente al enfoque que actualmente tienen las entidades culturales y los organismos que las apoyan. Un aspecto determinante en la economía creativa en los países en vía de desarrollo es su alto grado de informalidad, lo cual limita las ayudas y los apoyos que los gobiernos tradicionales puedan brindar, debido a las regulaciones propias de las políticas sectoriales. El Gobierno es un ente protagonista en los procesos de formalización de las ICC, y es quien debe liderar, junto con los artistas y creadores, el proceso de consolidación del sector, a fin de generar una infraestructura de largo plazo que permita su crecimiento $y$, por supuesto, la medición de su impacto.

Uno de los grandes retos para los responsables de formular políticas relacionadas con economías creativas es la obtención de datos e información confiable, ya que, dada la informalidad de los sectores que conforman la llamada economía creativa, la información disponible sobre estos no es suficiente y tampoco confiable para entender las dinámicas relacionadas con estos que se desarrollan en las ciudades y regiones. Por lo tanto, la información disponible no ayuda a generar hojas de ruta para el diseño de políticas sostenibles.

Todo lo anterior conlleva el reto de plantear y proponer indicadores económicos formales ajustados a la informalidad propia de las economías creativas. La economía creativa no debe generar falsas expectativas en torno a que se convierta en la única alternativa para superar problemas complejos y profundos, tales como la pobreza, el desempleo, la falta de educación - la desigualdad de oportunidades. Sin embargo, puede ser parte fundamental de las estrategias de una ciudad o región con relación a las problemáticas señaladas anteriormente. Con ello sí se generan los cambios necesarios y estructurales para que los sectores pertenecientes a las economías creativas no estén en desventajas respecto de los sectores económicos tradicionales.

\section{Referencias}

Acosta-Medina, J. K., Plata-Gómez, K. R., Puentes-Garzón, D. E. y Torres-Barreto, M. L. (2019). Influencia de los recursos y capacidades en los resultados financieros y en la competitividad empresarial: Una revisión de la literatura. I+ D Revista de Investigaciones, 13(1), 147157. https://doi.org/10.33304/revinv.v13n1-2019013

Banks, M. y Hesmondhalgh, D. (2009). Looking for Work in Creative Industries Policy. International Journal of Cultural Policy, 15(4), 415-430. https://doi. org/10.1080/10286630902923323

Benavente, J. M. y Grazzi, M. (2017). Politicas públicas para la creatividad y la innovación: Impulsando la economía naranja en América Latina y el Caribe. Banco Interamericano de Desarrollo. https://publi cations.iadb.org/publications/spanish/document/ Políticas-públicas-para-la-creatividad-y-la-innova ción-Impulsando-la-economía-naranja-en-Améric a-Latina-y-el-Caribe.pdf

Boix, R. y Lazzeretti, L. (2011). Las industrias creativas en España: una panorámica. Investigaciones Regionales, 22, 181-205.

Bustamante, E. (2009). De las industrias culturales al entretenimiento. La creatividad, la innovación. Viejos y nuevos señuelos para la investigación de la cultura. Revista Académica de la Federación Latinoamericana de Facultades de Comunicación Social, 78, 1-25.

Cámara de Comercio de Barranquilla. (2016). Las industrias creativas en el Atlántico: Mapeo cultural. Camara de Comercio de Bogotá; Centro de Información Empresarial (CIEB). http://hdl.handle. net/11520/18798 
David Juliao-Esparragoza, Mariangela Lopez-Lambraño, Alberto Muñoz-Santiago

Transición del concepto de industrias creativas y su papel en el entorno económico

Conferencia de las Naciones Unidas sobre Comercio y Desarrollo UNCTAC. (2010). Economía creativa: Informe 2010.

Cunningham, S. (2002). From Cultural to Creative Industries: Theory, Industry and Policy Implications. Media International Australia, 102(1), 54-65. https:// doi.org/10.1177/1329878X0210200107

Department for Digital, Culture, Media \& Sport. (1998). Creative Industries Mapping Documents 1998. Gobierno del Reino Unido. https://www.gov.uk/ government/publications/creative-industries-map ping-documents-1998

Department for Digital, Culture, Media \& Sport. (2001). Creative Industries Mapping Documents 2001-Foreword. https://www.gov.uk/government/ publications/creative-industries-mapping-docum ents-2001

Drake, G. (2003). 'This Place Gives Me Space': Place and Creativity in the Creative Industries. Geoforum, 34(4), 511-524. https://doi.org/10.1016/S0016-7185 (03)00029-0

Finlev, T., Maguire, R., Oppenheim, B. y Skvirsky, S. (2017). Future Landscapes of the Orange Economy: Creative Pathways for Improving Lives in Latin America and the Caribbean. https://publications.iadb.org/publ ications/english/document/Future-Landscapes-o f-the-Orange-Economy-Creative-Pathways-for-I mproving-Lives-in-Latin-America-and-the-Caribb ean.pdf

González Vélez, E. (2013). El ecosistema de las industrias culturales en colombia. Revista UIS Humanidades, 41(2), 81-103.

Hesmondhalgh, D. (2008). Cultural and Creative Industries. En T. Bennett y J. Frow (eds.), The SAGE Handbook of Cultural Analysis (pp. 552-569). SAGE Publications Ltd. https://doi.org/10.4135/9781848608443.n27

Howkins, J. (2002). The Creative Economy: How People Make Money from Ideas. Penguin UK.

Ministerio de Cultura de Colombia-Convenio Andrés Bello. (2003). Impacto económico de las industrias culturales en Colombia.

NU. CEPAL. (2014). Cultura y desarrollo económico en Iberoamérica. OEI.

O'Connor, J. (2010). The Cultural and Creative Industries: A Literature Review (2. ${ }^{\mathrm{a}}$ ed.). Creativity, Culture and Education.
Organización de las Naciones Unidas para la Educación la Ciencia y la Cultura UNESCO. (2010). Políticas para la creatividad. Guía para el desarrollo de las industrias culturales y creativas. Banco Interamericano de Desarrollo. https://es.unesco.org/creativity/sites/ creativity/files/220384s.pdf

Organización de las Naciones Unidas Para la Educación la Ciencia y la Cultura UNESCO y Confederación Internacional de Sociedades de Autores y compositores CISAC. (2015). Tiempos de cultura. EI primer mapa mundial de las industrias culturales y creativas. https://es.cisac.org/Universidad-CISAC/ Biblioteca/Estudios-y-guias/Tiempos-de-culturaEl-primer-mapa-mundial-de-las-Industrias-Cultur ales-y-Creativas

Organización Mundial de la Propiedad Intelectual OMPI. (2005). Industrias culturales y derecho de autor oportunidades y desafios. https://www.wipo.int/ edocs/mdocs/lac/es/ompi_da_mex_05/ompi_da_ mex_05_5.pdf

Podestá, P. y Hernández, M. C. (2012). Empresas de base cultural y creativa: Identificación y clasificación de sus factores críticos de desempeño (Culture and Creative Based Companies: Identifying and Classifying Their Critical Performance Factors). TEC Empresarial, 6(2), 17-27. https://doi.org/10.18845/ te.v6i2.526

Pose García, M. (2015). Economía creativa, dinámicas locales y gobernaza en entornos metropolitanos: El caso de la región metropolitana de Salvador de Bahia. DRD - Desenvolvimento Regional Em Debate, 5(2), 86-108. https://doi.org/10.24302/drd.v5i2.1007

Potts, J., Cunningham, S., Hartley, J. y Ormerod, P. (2008). Social Network Markets: A New Definition of the Creative Industries. Journal of Cultural Economics, 32(3), 167-185. https://doi.org/10.1007/s10824-0089066-y

Pratt, A. C. (2008). Creative Cities: The Cultural Industries and the Creative Class. Geografiska Annaler: Series B, Human Geography, 90(2), 107-117. https://doi. org/10.1111/j.1468-0467.2008.00281.x

Quartesan, A., Romis, M. y Lanzafame, F. (2007). Las industrias culturales en América Latina y el Caribe: Desafíos y oportunidades. https://publications. iadb.org/es/publicacion/15194/las-industrias-cult urales-en-america-latina-y-el-caribe-desafios-yoportunidades

Rotondo, A. S. y Legonía Córdova, E. (2018). Crecimiento con desigualdad: Brechas y retos de la economía de las industrias culturales y creativas del Perú. En J. J. Sánchez Balaguer, S. Arroyo Serrano, J. F. Parra Azor 
y A. J. Verdú Jover (coords.), Las industrias culturales y creativas en Iberoamerica. Evolución y perspectivas (pp. 347-366). Tadigra.

Shane, S. (1993). Cultural Influences on National Rates of Innovation. Journal of Business Venturing, 8(1), 59-73. https://doi.org/10.1016/0883-9026(93)90011-S

Szpilbarg, D. y Saferstein, E. A. (2014). De la industria cultural a las industrias creativas: Un análisis de la transformación del término y sus usos contemporáneos. Estudios de Filosofía Práctica e Historia de las Ideas, 16(2), 99-112.

Tian, M., Deng, P., Zhang, Y. y Salmador, M. P. (2018). How Does Culture Influence Innovation? A Systematic Literature Review. Management Decision, 56(5), 1088-1107. https://doi.org/10.1108/MD-05-20170462

Verspagen, B. (2006). University Research, Intellectual Property Rights and European Innovation Systems. Journal of Economic Surveys, 20(4), 607-632. https:// doi.org/10.1111/j.1467-6419.2006.00261.x 